\title{
Features of Meditation Apps: A Scoping Review
}

\author{
Raghad MUHIYADDIN ${ }^{\mathrm{a}}$, Alaa ABD-ALRAZAQ ${ }^{\mathrm{a}}$, Mohannad ALAJLANI ${ }^{\mathrm{b}}$, Zubair \\ SHAH $^{\mathrm{a}}$, Tanvir ALAM ${ }^{\mathrm{a}}$ and Mowafa HOUSEH ${ }^{\mathrm{a}, 1}$ \\ ${ }^{a}$ College of Science and Engineering, Hamad Bin Khalifa University, Doha, Qatar \\ ${ }^{\mathrm{b}}$ Institute of Digital Healthcare, WMG, University of Warwick, Warwick, United
}

Kingdom

\begin{abstract}
This review aims to provide an overview of the features of meditation apps as described in empirical literature. Nine databases were searched for this review. Search terms were related to all types of meditation. Study selection and data extraction of the included studies were conducted by two reviewers. We included 93 studies in this review. Headspace was the most common app among studies and the most common type of meditation was mindfulness. Stress was the most targeted health condition by the studies. Future research needs to focus on different mental conditions other than stress to understand the effect of meditation apps on mental health.
\end{abstract}

Keywords. Meditation, mindfulness, mobile applications, scoping review

\section{Introduction}

Meditation is a well-known practice that helps in reducing stress, depression, anxiety, and other mental disorders [1]. It is the process of an individual focusing their awareness to relax the mind and body [2]. In the past decade, meditation has become accessible ditially. People can access meditation applications through their phones, tablets, or computers. The main advantage of online-based meditation programs is convenience because of their ubiquitousness and low cost. There are many meditation apps on the market, including well-known apps such as Headspace, Calm, and Insight Timer. However, most studies conducted investigating these meditation apps focus on mental disorders. No scoping review has explore and summarize the features of meditation apps, despite the importance of this information. To fill this gap, this review aims to provide an overview of the features of meditation apps as described in the empirical literature.

\section{Methods}

The guidelines of the Joanna Briggs Institute (JBI) methodology were followed to conduct this scoping review [3]. Nine bibliographic databases were searched for this review: MEDLINE, EMBASE, PsycINFO, CINAHL, Scopus, IEEE Xplore, Cochrane

\footnotetext{
${ }^{1}$ Corresponding Author: Dr. Mowafa Househ, College of Science and Engineering, Hamad Bin Khalifa University, Doha, Qatar; Email: mhouseh@hbku.edu.qa.
} 
Library, Google Scholar, and ACM Digital Library. Because Google Scholar recommended thousands of results, only the first 100 studies were included. Search terms that were chosen are based on the intervention (meditation) and the platform (mobile applications). The search strings used to search all of the above databases can be found in Appendix 1. No restrictions were applied on population (i.e., users of the app), measured outcome, setting, country of publication, and year of publication. The study selection process consisted of screening titles and abstracts of all retrieved studies, then reading full texts of the remaining studies. Data were extracted from the included studies using a data extraction form created in Excel (Appendix 2). The study selection and data extraction processes were carried out by two reviewers (RM \& MA) independently. A third reviewer (AA) was consulted in cases of disagreement. Extracted data were summarized using two tables and narratively described.

\section{Results}

\subsection{Search Results}

As shown in Appendix 3, 1,201 studies were retrieved from searching the nine databases, and 342 duplicates were removed before starting the study selection process. After scanning the titles and abstracts of the 859 remaining studies, 748 studies were excluded studies due to irrelevant intervention, irrelevant platform, type of publication, and nonEnglish studies. Reading the full text of the remained 111 studies led to excluding 18 studies due to the irrelevant intervention, irrelevant publication type, unavailable full text, and non-English studies. Overall, 93 studies were included in the data synthesis. Appendix 4 shows the list of all included studies.

\subsection{Characteristics of Studies}

As seen in Appendix 5, most studies included were journal articles $(n=77,82.8 \%)$. The included studies were published in 22 different countries; however, about two-thirds of the studies were published in the United States $(n=61,66 \%)$. The majority of studies were conducted between 2017 and 2019 ( $n=80,86 \%$ ). About 63\% of studies had a sample size of less than $100(\mathrm{n}=59)$, while the remaining studies had a sample size of 100 or more $(n=33,35.5 \%)$. The mean age of participants was only reported in 70 studies, in which the average was 35.2 yrs. Only 85 studies reported the sex of the participants, where $25.8 \%$ of participants were males. Almost half of the participants were recruited from the community $(n=45,48.4 \%)$. Appendix 6 shows the characteristics of each included study.

\subsection{Features of Meditation Apps}

As shown in Appendix 7, 50 meditation apps were mentioned in the 93 studies included in this review. The most common app used in the included studies was Headspace $(n=17)$ followed by Calm $(n=9)$, AEON $(n=4)$, and Pacifica $(n=4)$. The studies' featured meditation apps were used for 16 different health conditions. The most common health condition targeted by the meditation apps was stress $(n=39)$, followed by mental health $(n=8)$, depression $(n=7)$, and cancer $(n=7)$. There were other health conditions targeted 
such as nicotine dependence $(n=3)$, essential hypertension, and dementia $(n=1)$ (Appendix 7). We identified six different types of meditation delivered by the apps. Mindfulness meditation was the most common type $(n=55)$ followed by guided meditation ( $n=19)$, and Mindfulness-Based Stress Reduction (MBSR) $(n=8)$. The majority of apps in the included studies could be operated on both Android as well as iOS $(n=72)$ devices. The majority of the reported apps were in English only $(n=29)$ or multi-language $(n=30)$. Some apps were developed in non-English languages $(n=11)$. More than half of the apps were still available in the app store at the time of this study $(n=60)$. Some were unavailable $(n=24)$, while the availability status for some were unknown because the studies did not mention the app used $(n=9)$. As for the cost, 35 apps were free with in-app purchases, 23 apps were completely free, while the costs of 32 apps are unknown. Appendix 8 highlights the characteristics of all included meditation apps.

\section{Discussion}

\subsection{Principal Findings}

This scoping review aimed to report the features of meditation apps as reported in the empirical literature. In the 93 included studies, around 50 different apps were mentioned. The top three apps mentioned were Headspace, Calm, and AEON. Headspace is currently the leading app in the market due to many factors such as ease of use, variety of exercises offered, reminders, aesthetics, and being scientifically supported [4]. Although meditation apps were used for various health conditions, they were used mainly for alleviating stress. It is known that meditation has a substantial impact on reducing stress, other health conditions have yet to be targeted. This allows room for improvement for future app developers or conducting future research on different populations with health conditions. Additionally, only one app targeted people with dementia, but more studies discussing meditation's impact on dementia should be conducted, as it has been proven that meditation can help with improving neural markers and improving working memory [5]. The most common type of meditation reported in studies was mindfulness meditation, followed by guided meditation.

\subsection{Strengths \& Limitations}

This review encaptures a large number of studies that explore app features and the effect they have on different health conditions. This review is considered to be inclusive for a few reasons. Firstly, it included 93 studies, which can be considered comprehensive. Secondly, nine major databases were searched. Thirdly, no restrictions were applied on type of meditation, target health condition, population, measured outcome, year of publication, setting, country of publication. In addition, the study selection and data extraction processes were done by two reviewers, which reduces the selection bias.

Although this review was inclusive in many ways, it was at times exclusive. There were many types of meditation-related activities that were excluded such as yogic practices, religious practices, martial-arts training, and exercise programs. Additionally, only studies written in English were included, thereby, we might have missed some studies. We also only included empirical studies with a certain type of publications such as peer-reviewed articles, thesis, conference proceedings. Lastly, we did not assess the 
effectiveness and safety of using meditation apps, and, to the best of our knowledge, no previous study investigated this. Therefore, systematic reviews are needed to evaluate the effectiveness and safety of using meditation apps.

\subsection{Research \& Practical Implications}

The majority of the included studies were conducted in developed countries. Future research should be conducted in developing countries, where people suffer more from mental health issues and have insufficient resources to combat them (e.g., psychiatrists) [6-8]. Additionally, further research should be conducted on health conditions other than stress. Because mindfulness meditation was mentioned in more than half of the studies, we encourage researchers to explore types of meditation apps for future research. Specific guidelines should be followed when conducting future research as there was inconsistency in many of the population (e.g., the mean age, and sex) and meditation apps (e.g., language of the app and the cost) characteristics reported. Lastly, app developers should build multi-language meditation apps to grow a user base, rather than limiting their app to one language or region in which users can access the app.

\section{Conclusions}

This study identified and explored 50 different meditation apps. Some of the most popular apps were Headspace, Calm, Aeon, Pacifica, Craving to Quit, and Situated Interactive Mindfulness App. Although there were 16 health conditions identified, stress was the most targeted health condition. Future research should focus on health conditions other than stress to be able to understand the effect of meditation on many different mental health conditions. Appendix files are available at GitHub: https://github.com/rmuhiyaddin/Appendix.git.

\section{References}

[1] Schreiner I, Malcolm JP. The benefits of mindfulness meditation: Changes in emotional states of depression, anxiety, and stress. Behaviour Change. 2008 Sep;25(3):156-68.

[2] Edenfield TM, Saeed SA. An update on mindfulness meditation as a self-help treatment for anxiety and depression. Psychology research and behavior management. 2012;5:131.

[3] Peters MD, Godfrey C, McInerney P, Munn Z, Tricco AC, Khalil HA, Munn Z. Chapter 11: Scoping reviews. JBI Manual for Evidence Synthesis [Internet]. Adelaide: JBI, 2020 [cited 2019 Aug 14]. doi.org/10.46658/JBIMES-20-12.

[4] PsyberGuide. Professional Review of the App Headspace. One Mind PsyberGuide. 10 Dec. 2020. onemindpsyberguide.org/expert-review/headspace-professional-review.

[5] Abbasi J. Meditation App Improves Attention in Young Adults. JAMA. 2019;322(6):495. Crossref, doi:10.1001/jama.2019.11649.

[6] Murray CJ, Vos T, Lozano R, Naghavi M, Flaxman AD, Michaud C, Ezzati M, Shibuya K, Salomon JA, Abdalla S, Aboyans V. Disability-adjusted life years (DALYs) for 291 diseases and injuries in 21 regions, 1990-2010: a systematic analysis for the Global Burden of Disease Study 2010. The lancet. 2012 Dec 15;380(9859):2197-223.

[7] Oladeji BD, Gureje O. Brain drain: a challenge to global mental health. BJPsych international. 2016 Aug;13(3):61-3.

[8] Anthes E. Mental Health: There's an App for That. Nature. 2016;532(7597):20-23. Crossref, doi:10.1038/532020a. 